\title{
The localization of strain and $c$-axis evolution in anisotropic ice
}

\author{
Christopher J. L. WILSON, Hadi M. SIM \\ School of Earth Sciences, University of Melbourne, Parkville, Victoria 3010, Australia \\ E-mail:cjlw@unimelb.edu.au
}

\begin{abstract}
Using a series of combined compression-simple-shear experiments, it has been possible to investigate strain distributions and crystal-orientation fabrics related to varying layer orientation in ice. A variety of flattening strains accompanied by layer buckling, simple shear and the development of a lenticular layering are produced in anisotropic ice masses. In samples where the creep curve has only just reached a minimum strain rate, the $c$-axis preferred orientation is similar to that in the starting material, with specific $c$-axis concentrations affected by the extent of preserved host grains. At shear strains where $\gamma \leq 1$, it was found that the $c$-axis preferred orientations were highly variable depending on the magnitude of strain, strain distribution and upon the modification and degree of rotation of initial $c$-axis preferred orientation. However, once recrystallization dominates in high-strain zones $(\gamma \geq 1)$, there is a rapid development of an asymmetric two-maxima fabric with little evidence of any contributions from inherited fabric elements. The final $c$-axis pattern is asymmetric with respect to the direction of shortening, with a strong maximum at $\sim 80^{\circ}$ to the shear zone, with a sense of asymmetry in the direction of the shear, and a secondary maximum lying at $\sim 50^{\circ}$ to the plane of shearing.
\end{abstract}

\section{INTRODUCTION}

It is known that ice near the bedrock and within ice sheets may be layered and highly anisotropic, with a well-defined stratigraphic layering and variable crystallographic orientations. This may be an outcome of a combination of accumulation record, resulting in a primary layering, and deformation history. However, this ice stratigraphy is often disturbed near the margins of a glacier (Glasser and others, 1998; Marmo and Wilson, 1998), at the front of ice sheets (Tison and others, 1998) and in areas such as ice divides (Castelnau and others, 1998). Such perturbations will cause local rotation of the layering into structures such as shear zones, recumbent folds and attenuated fold limbs (Hambrey, 1977; Hudleston, 1980). In such an environment the anisotropic ice mass still experiences essentially the same bulk stress while undergoing longitudinal flow. However, variations in the orientation of primary layering and crystallographic variability are thought

Table 1. Plane-strain compression experiments undertaken at $-1^{\circ}$ C. Compressive stress $(\sigma)$, shortening $\%\left(\varepsilon_{1}\right)$ and bulk strain rate $\left(\dot{\varepsilon}_{\mathrm{f}} \times 10^{-8} \mathrm{~s}^{-1}\right)$

\begin{tabular}{|c|c|c|c|c|}
\hline Layer orientation & $\begin{array}{l}\text { Experiment } \\
\text { No. }\end{array}$ & $\begin{array}{l}\text { Initial (final) } \\
\text { axial stress } \sigma \\
\\
\mathrm{MPa}\end{array}$ & $\begin{array}{l}\varepsilon_{1} \\
\%\end{array}$ & $\begin{array}{c}\text { Bulk } \\
\text { strain rate } \\
10^{-8} \mathrm{~s}^{-1}\end{array}$ \\
\hline $\begin{array}{l}\text { Rotated } 15^{\circ} \text { to } x_{2} x_{3} \\
\text { about } x_{2} \text { axis }\end{array}$ & $1-13$ & $0.64(0.52)$ & 9.1 & 9.9 \\
\hline $\begin{array}{l}\text { Rotated } 45^{\circ} \text { to } x_{2} x_{3} \\
\text { about } x_{2} \text { axis }\end{array}$ & $1-19$ & $\begin{array}{l}0.38 \text { ( } 0.28 \text { top, } 0.34 \\
\text { bottom surface) }\end{array}$ & 29.3 & 5.5 \\
\hline Parallel to $x_{2} x_{3}$ & $1-21$ & $0.64(0.52)$ & 15 & 2.7 \\
\hline
\end{tabular}

to play an important role in controlling rates and the nature of the ice mass deformation (Hudleston, 1980).

Tight observational constraints as to what happens to the layering and the local strain geometry are rarely available in nature, although it is often assumed that they correspond to simple shear. This paper describes a series of deformation experiments on ice aggregates that contain a layering consisting of a strong initial grain-shape anisotropy and a known initial $c$-axis crystallographic preferred orientation. The effect of this layering and $c$-axis preferred orientation on the nature of the final fabric and strain distribution was investigated using plane-strain experiments (Table 1) and a combination of unconfined axial compression and simple shear

Table 2. Summary of combined compression and shear experiments undertaken at $-2^{\circ} \mathrm{C}$ with a compressive stress of $0.22 \mathrm{MPa}$ and shear stress of $0.4 \mathrm{MPa}$. Bulk shortening \%

$\left(\varepsilon_{1}\right)$, shear strain $(\gamma)$, minimum strain rate in compression $\left(\dot{\varepsilon}_{\mathrm{m}} \times 10^{-8} s^{-1}\right)$ and final strain rate $\left(\dot{\varepsilon}_{\mathrm{f}} \times 10^{-8} s^{-1}\right)$

\begin{tabular}{lccccc}
\hline Layer orientation & $\begin{array}{c}\text { Experiment } \\
\text { No. }\end{array}$ & $\varepsilon_{1}$ & $\gamma$ & $\dot{\varepsilon}_{\mathrm{m}}$ & $\dot{\varepsilon}_{\mathrm{f}}$ \\
& & $\%$ & & $10^{-8} \mathrm{~s}^{-1}$ & $10^{-8} \mathrm{~s}^{-1}$ \\
\hline Parallel to $x_{1} x_{2}$ & $2-27$ & 0.02 & 0.03 & 8.8 & 8.8 \\
Parallel to $x_{1} x_{2}$ & $2-29$ & 0.4 & 0.09 & 11 & 12 \\
Parallel to $x_{1} x_{2}$ & $2-32$ & 3.2 & 1.14 & 17 & 17 \\
Parallel to $x_{1} x_{2}$ & $2-36$ & 2.8 & 0.5 & 7.2 & 12 \\
Parallel to $x_{1} x_{2}$ & $2-40$ & 8.34 & 1.43 & 5.9 & 38 \\
Parallel to $x_{1} x_{2}$ & $2-41$ & 8.67 & 2.75 & 11 & 42 \\
Rotated $20^{\circ}$ to $x_{1} x_{2}$ & $2-43$ & 12.1 & 1.52 & 3.9 & 39 \\
$\quad$ about $x_{2}$ axis & & & & & \\
Rotated $20^{\circ}$ to $x_{1} x_{2}$ & $2-44$ & 10.97 & 1.07 & 7.8 & 35 \\
$\quad$ about $x_{2}$ axis & & & & & \\
\end{tabular}



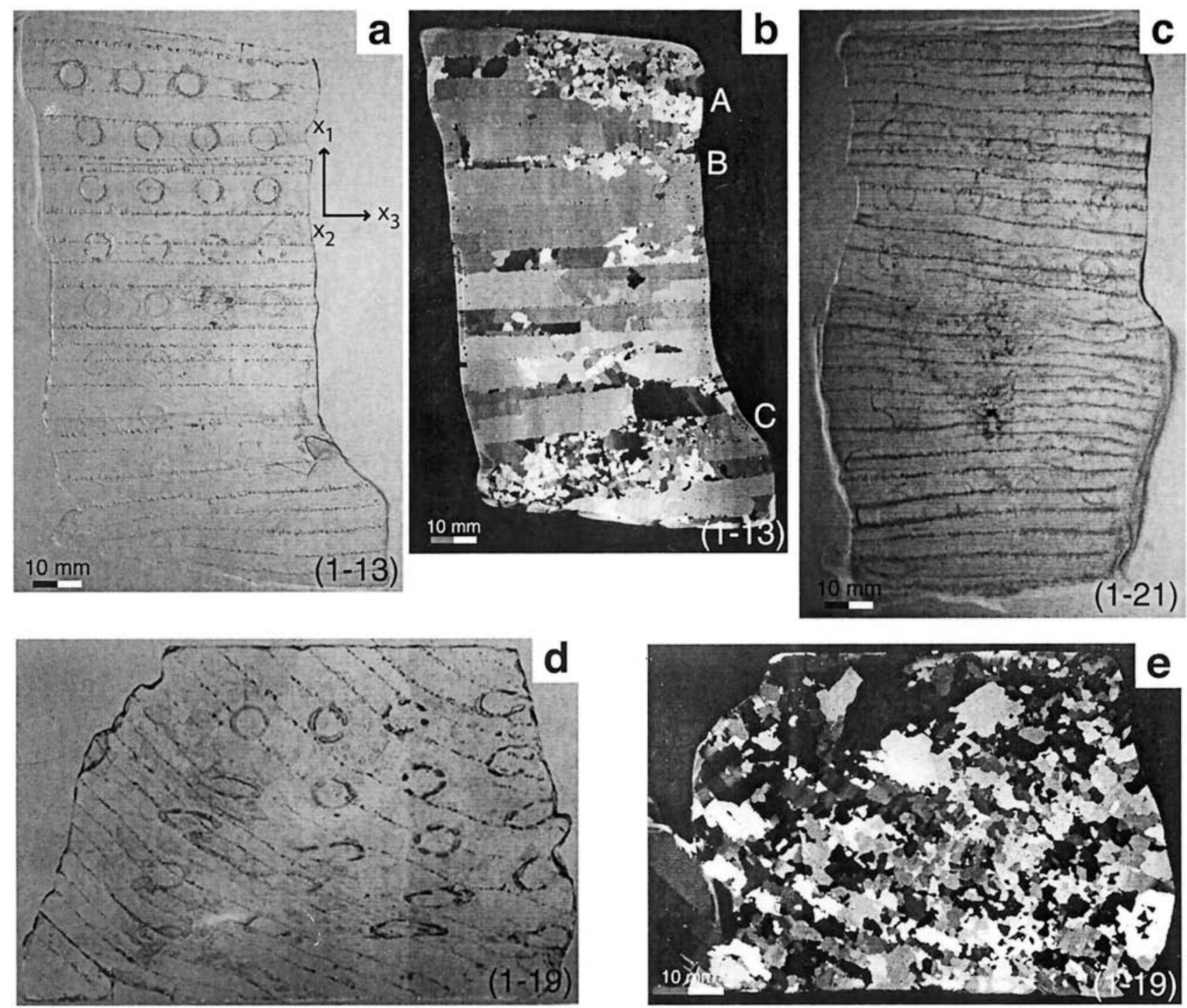

Fig. 1. Structural changes associated with deformation of the layered ice at $-1^{\circ} \mathrm{C}$. (a) Thick section, parallel to $x_{1} x_{3}$, of experiment 1-13 in plane polarized light, showing the nature of the initial layers and deformation of initially circular strain markers. The initial layering was gently inclined to the $x_{2} x_{3}$ plane but rotated $15^{\circ}$ about the $x_{2}$ axis. (b) Thin section between crossed polarizers of experiment 1-13, showing undeformed regions and three areas $(A-C)$ where deformation and significant recrystallization has occurred. (c) Thick section (1 mm), parallel to $x_{1} x_{3}$, of experiment 1-21 in plane polarized light, showing buckled layering and distribution of strain as identified by the deformation of a set of initially circular strain markers. The initial layering was parallel to $x_{2} x_{3}$. (d) Thick $x_{1} x_{3}$ section of experiment 1-19 in plane polarized light, showing the initial layers and deformation of initially circular strain markers. (e) $x_{1} x_{3}$ section of experiment 1-19, between crossed polarizers, with substantial recrystallization occurring in regions of higher strain.

(Table 2). This was achieved by varying the initial orientation of the anisotropy and the crystallographic preferred orientation patterns with respect to the bulk shortening $\left(x_{1}\right)$ and extension $\left(x_{3}\right)$ axes (Figs 1-3a). In the plane-strain deformation experiments, there was confinement of the sample parallel to the $\left(x_{2}\right)$ axis. The resulting bulk strains in these directions are $\varepsilon_{1}<\varepsilon_{2}=0<\varepsilon_{3}$ and are expressed as a percentage shortening. It will be shown that the orientation of the layering, and the differing fabrics of the ice have little effect on the strain response of the grain aggregate and, consequently, on the strength. In the process, the ice rapidly recrystallizes in high-strain zones and preserves little memory of the earlier fabrics.

\section{EXPERIMENTAL AND STRAIN ANALYSIS METHODS}

An initial set of $-1{ }^{\circ} \mathrm{C}$ experiments (Table 1) was undertaken on an apparatus described by Wilson (1981) in order to estab- lish how strain could be distributed across a layered ice sample (Figs 1 and 2). The majority of these results are described inWilson (2000) and will not be elaborated on here, but served as the basis for undertaking the $-2{ }^{\circ} \mathrm{C}$ experiments described herein. The experiments involved the use of samples comprising layers ( $\sim 10 \mathrm{~mm}$ wide) of fine-grained ice intergrown with elongate crystals, similar to multilayered columnar ice (Gold, 1972). The layered ice was prepared from distilled and de-aerated water according to previously described methods (Gold, 1972; Wilson, 1981). It has a marked tendency to grow more readily in directions perpendicular to the $c$ axis than parallel to it. Because of this, grains with their $c$ axes perpendicular to the layering tend to grow preferentially parallel to the layering, at the expense of smaller variably oriented grains. These finer grains with diameters of $\sim 3 \mathrm{~mm}$ are isolated within or between the elongate grains that define the layering. Layered samples were machined into rectangular blocks measuring approximately $75 \mathrm{~mm} \times 50 \mathrm{~mm} \times 50 \mathrm{~mm}$.

Estimates of the orientation and magnitude of the local 


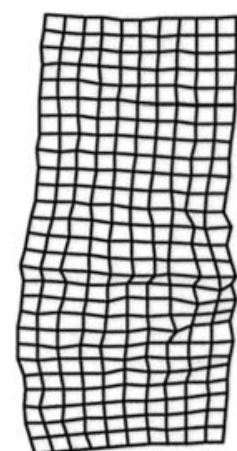

a. Deformed grid

$\varepsilon_{1} \approx 10 \%$ shortening
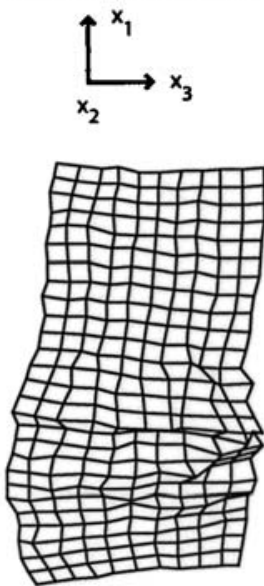

b. Deformed grid

$\varepsilon_{1} \approx 14 \%$ shortening

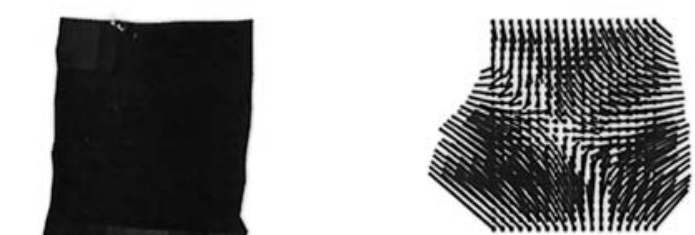

c. Displacement vectors $\varepsilon_{1} \approx 30 \%$ shortening.

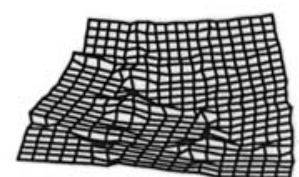

d. Deformed grid $\varepsilon_{1} \approx 30 \%$ shortening.

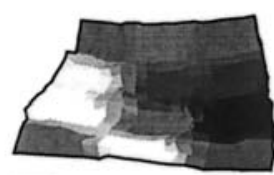

-0.3
-0.15

Shear strain

$(\gamma)$
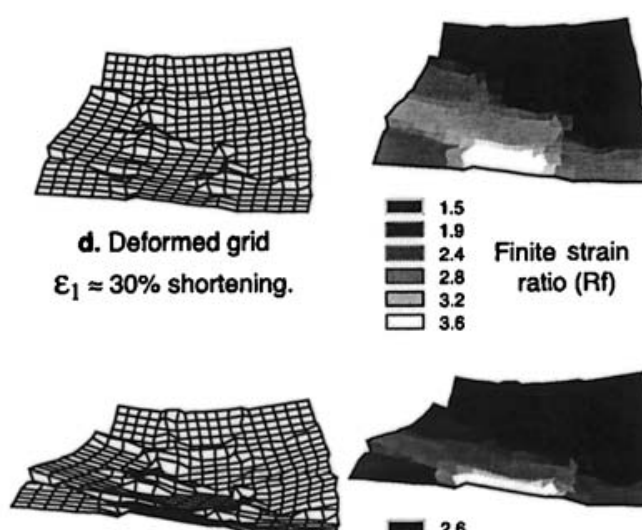

๑. Deformed grid

$\varepsilon_{1} \approx 38 \%$ shortening.

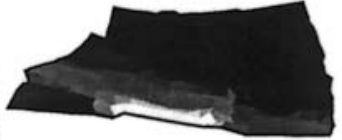

2.6
4.1

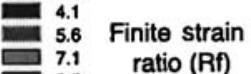
8.5 ratio (Rf)

Experiment 1-19

\section{Experiment 1-21}

Fig. 2. Finite strain in experiments 1-21 and 1-19. (a) Deformation grid and contour plots of $\mathrm{Rf}$ after $10 \%$ shortening $\left(\varepsilon_{1}\right)$ in experiment 1-21, determined from the strain markers seen in Figure 1c. Rf is $\geq 1$ (undeformed state). (b) Grid deformed a further $4 \%$ showing how strain continues to be localized in the initial zone of shearing. (c) Displacement vectors of markers after $10 \%$ bulk shortening in experiment 1-19, and contour plots of shear strain. (d) Deformation grid and contour plots of $\mathrm{Rf}$ after $30 \%$ bulk shortening established from using the strain markers seen in Figure 1d. (e) Grid deformed a further 8\%, showing how strain continues to be localized in the initial zone of shearing.

principal strains were obtained from the deformed shape of $5 \mathrm{~mm}$ diameter circles, on $10 \mathrm{~mm}$ centres, inscribed on faces that parallel the $x_{1} x_{3}$ strain axes. These markers were well preserved at low strains but became difficult to identify at higher strains; upon deformation they become strain ellipses. The strain ellipses show the magnitude of finite strain and the direction of the principal stretching directions (Fig. 2). They allowed us to calculate the final ellipticity $\mathrm{Rf}$ value (Lisle, 1985; Twiss and Moores, 1992) that utilizes the systematic shape changes that occur in deformed ellipsoidal objects. This is best displayed in the form of $\mathrm{Rf}$ contour plots (Bons and others, 1993) and as a set of displacement vectors that demonstrate the local flow pattern in the material (Fig. 2). The technique is based on calculating the theoretical distribution of final ellipticities and orientations that result from imposing different strains on the circles of known spacing and orientation. The two-dimensional deformation analysis software "Marker Analysis" (Bons and others, 1993) was used to analyze quantitatively the kinematics of the deformation on the $x_{1} x_{3}$ face (Fig. 1) using the preserved markers. This analysis was only possible on samples deformed in plane strain (Table 1), whereas markers used in axial compression tests (Table 2) were rarely preserved on the unconstrained $x_{1} x_{3}$ face. Displacement vectors have been resolved from the centre of each sample using the position of the inscribed strain markers in the undeformed sample and comparing these positions with their locations after deformation (Fig. 2).

In each of the experiments described in Table 1 there is a high degree of anisotropic deformation in individual portions of the sample (Fig. 1). Therefore the final axial stress (Table 1) distribution within an individual sample is variable. As pointed out by Wilson (2000), the macroscopic (bulk) strain rate in each of the individual experiments is a combination of effects related to the variations in the initial orientation of the anisotropy, the crystallographic preferred orientation patterns and relationship to the bulk shortening $\left(x_{1}\right)$ and extension $\left(x_{3}\right)$ axes, rather than the simple relationship between rheology and $c$-axis crystallographic fabric that has been suggested by Castelnau and others (1998). In the experiments illustrated in Figure 1, it is difficult to link to the microscopic behaviour of individual areas in the sample, as the stresses and strain rates are different in each individual grain.

The majority of tests described in the present paper were undertaken using deformation apparatuses and conditions similar to those described by $\mathrm{Li}$ and others (1996) and were performed under both constant stress and constant shear 
strain-rate conditions at $-2{ }^{\circ} \mathrm{C}$ (Table 2). In this study, the mechanical behaviour of variously oriented samples of anisotropic ice is compared to the behaviour of the isotropic samples in uniaxial compression tests (e.g. Li and others, 1996; Li and Jacka, 1998). The ice-test sample, measuring approximately $70 \mathrm{~mm} \times 55 \mathrm{~mm} \times 37 \mathrm{~mm}$, was frozen into two support boxes. The strain is not uniformly distributed in the sample but is localized into a central zone of higher shear strain (Fig. 3a and zone 3 Fig. 4a).

The tests were undertaken inside a freezer box, with the controls and recording system outside it as described by Li and others (1996). The temperature was controlled by circulating silicon oil around a sample shielded by a film of aluminium foil. Thermistors were placed adjacent to the specimen and at selected sites in the silicon oil bath to record the temperature of the test. The temperature variation was never more than $\pm 0.2^{\circ} \mathrm{C}$ during the month-long experiments. Orientation of $c$ axes was obtained using the fabric analysis apparatus that has been illustrated by Wilson and others (in press). Microstructural images were acquired using a digital camera. They were typically on the order of $2000 \times 2000$ pixels that were decomposed and reprocessed as axial-distribution diagrams or AVA diagrams (Achsenverteilungsanalysis) or as scatter diagrams of individual grain $c$-axis orientation plotted on an equal-area stereographic net. A standard colour code (Fig. 3c) was used to identify the $c$-axis directions as azimuths and dips in the micrographs. In this scheme, north-trending axes are in blue, east-trending axes are in orange, axes normal to the plane of the thin section are in black, and gradational shades represent angular differences between neighbouring pixels. A change in colour intensity to the centre of the colour circle (black) corresponds to $c$ axes increasing in plunge from horizontal, on the periphery, to vertical at the centre of the net.

\section{PROPAGATION OF STRAIN}

In the experiments described herein, it is possible to consider four scenarios. Cases 1 and 2 relate to a plane-strain compression, and cases 3 and 4 relate to a deformation regime that involves combined compression and simple shear. These four scenarios are:

\section{Case 1}

When the flattening strain is sub-parallel to the layering in an ice mass, local deformation instabilities may originate in this plane. For instance, in the deformed blocks in Figure $1 \mathrm{a}-\mathrm{c}$ the deformation is heterogeneously distributed throughout the sample. This is particularly obvious in experiment 1-13 (Fig. la and b). Here the layering was initially misoriented to $x_{2} x_{3}$ by $15^{\circ}$ to the plane of flattening, and the deformation became partitioned into selected sections of the layering. These areas of strain accumulation are always accompanied by recrystallization (Fig. 1b), and little strain is observed in the adjacent regions, which preserve their undeformed microstructure (Fig. lb). The magnitude of the finite strain (Rf) required to induce such recrystallization is very small, with Rf contour values of $\geq 2$ (Fig. $2 \mathrm{a}$ ). With the continuation of flattening (Fig. 2b), the propagation of strain and recrystallization tends to be confined to these lens-shaped areas and not uniformly distributed throughout the sample.

\section{Case 2}

In a situation where the layering is inclined at a high angle to a flattening plane (Fig. ld and e) there is bending of the layering over a broad region and no obvious strain localization. The bulk deformation is reflected in the material displacement vectors (Fig. 2c) of individual areas. Both positive and negative shear strain values reflect the sense of shear across the sample, with the orientation of the major principal axes of strain ellipses (Fig. ld) reflecting the change in the amount of shear strain. Using the centre-to-centre point distribution of the strain markers, it is possible to define a deformation grid (Fig. 2d) and related Rf values. The contour plot of $\mathrm{Rf}$ (Fig. 2d) shows an increase towards the base of the sample, with values of $<2.8$ in areas where there is extensive recrystallization (Fig. le). If this grid is further deformed (Fig. 2e) then correspondingly higher strain values are generated.

\section{Case 3}

This relates to the application of two orthogonal strains, namely, compression parallel and simple shear perpendicular to the layering (Figs 3 and 4). In this situation, the ice begins to buckle and is deformed at a higher rate in a central shear zone (Fig. 3a). At small strains $(\gamma<0.1$, Fig. 3d; stage 1$)$ there is always minor migration of pre-existing grain boundaries, particularly in the smaller-sized grain population, and the preservation of the initial $c$-axis fabrics in the larger elongate grains (Fig. 3d). The preservation of this earlier fabric is clearly seen as a concentration of highly oriented grains $(80-$ $90^{\circ}$ ) in the histogram (Fig. 3d) as a strong deviation from a theoretical uniform crystal-orientation pattern. These histograms show the frequency of angles between the $c$ axes of adjacent grains, and superimposed on the histogram is a curve showing the theoretical distribution between two $c$ axes, one of fixed and one of random orientation (Jacka and Maccagnan, 1984). As the aggregate deforms, the layering is overprinted by numerous recrystallized grains (Fig. 3f and g; stage 2) that relate to the initiation of buckling in the layers, best seen in vertical sections (Fig. 4). With the amplification of the buckles (Fig. 4), dynamic recrystallization is accelerated and there is extensive new grain development. Strain is localized into discrete zones of recrystallization (Fig. 4c-f, zone 3) that parallel the flow plane. The zone of new recrystallized grains is bounded by a transitional zone (Fig. 4c and e, zone 2) and domains preserving pre-existing grain structures (Fig. 4c and e, zone 1).

\section{Case 4}

Where layering is inclined to the compression direction, the initially continuous ice layering is thinned in the zone of high shear strain, often in a lenticular fashion (Fig. 5, zone 3 experiment 2-43 vertical section). Where shortenings $(\varepsilon)$ exceed $3 \%$ and shear strains $(\gamma)$ are $>$, i.e. when layering rotation is $>45^{\circ}$, there is no evidence of the pre-existing grain population, and the sample is extensively recrystallized in areas that experience high shear strains (Fig. 5, zone 3).

\section{EVOLUTION OF $c$-AXIS PREFERRED ORIENTATION}

Orientations of ice $c$ axes have been obtained from horizontal thin sections cut parallel to $x_{2} x_{3}$ (Figs $\left.3-5\right)$ and vertical thin sections cut parallel to $x_{1} x_{3}$ (Figs 4 and 5). Two stages in the early evolution of the fabric were investigated (Fig. 3). The 
(a)

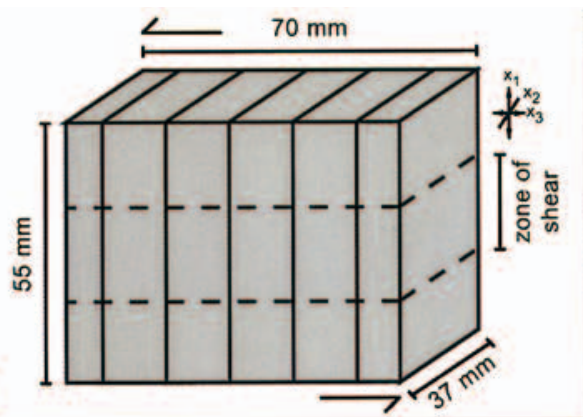

(c)

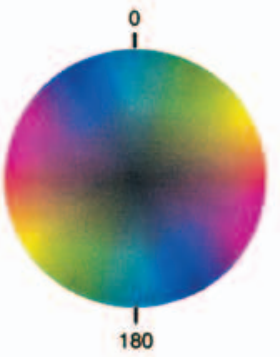

Initial layering parallel to $x_{1} x_{2}$ plane Compressive stress Shear stress

Temperature

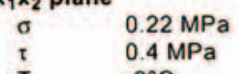

$-2^{\circ} \mathrm{C}$

\section{Stage 1}

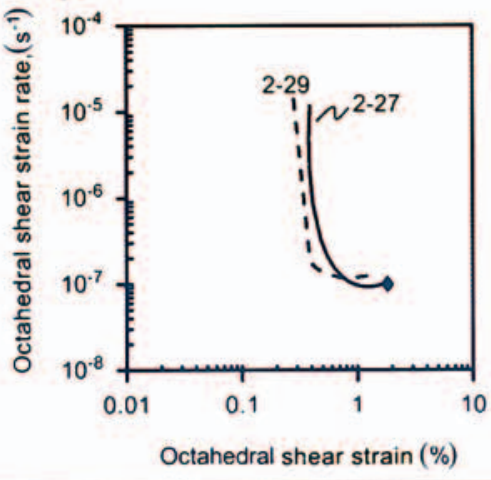

(d)

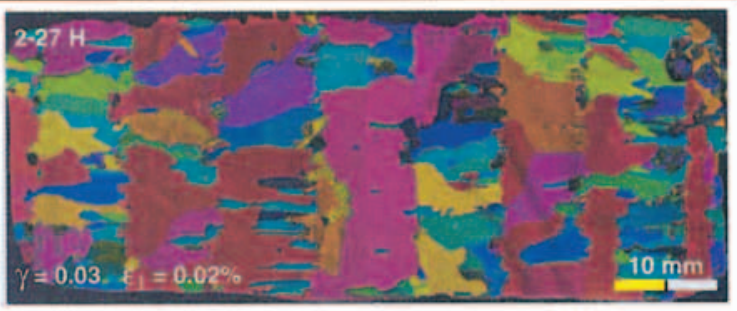

Horizontal section $\left(x_{2} x_{3}\right)$ experiment $2-27$
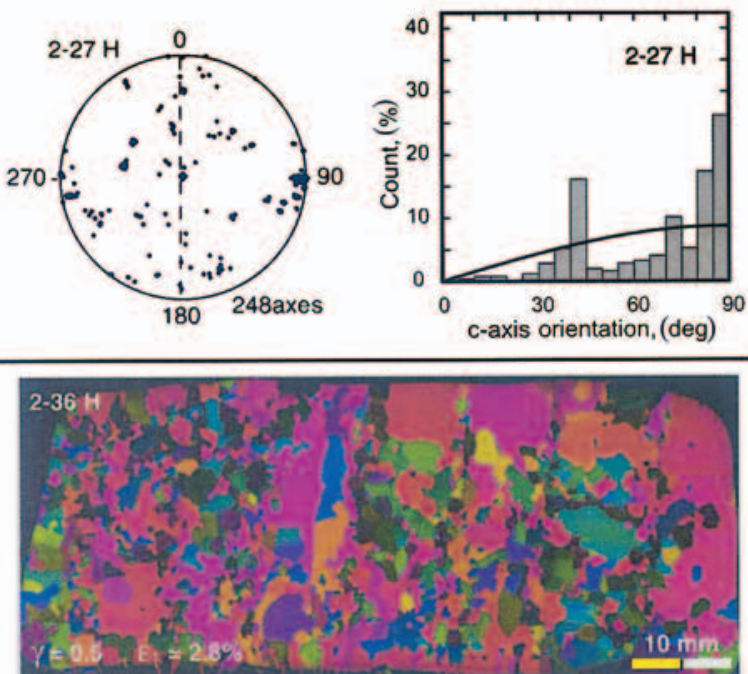

(f) Horizontal section $\left(x_{2} x_{3}\right)$ experiment 2-36
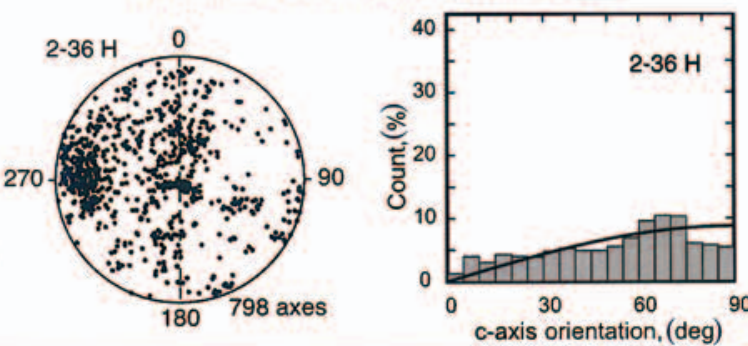

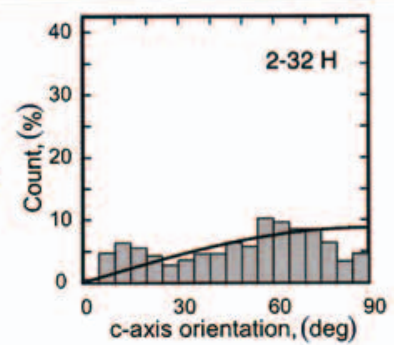

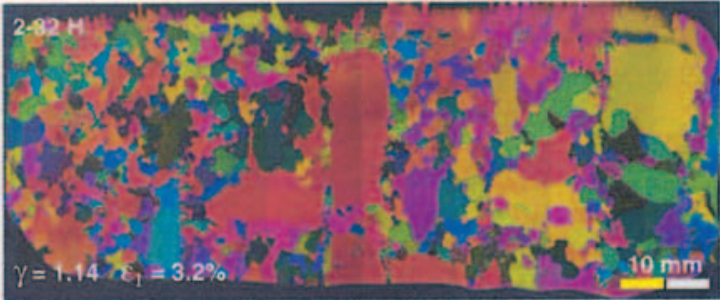

Horizontal section $\left(x_{2} x_{3}\right)$ experiment 2-32

Fig. 3. Experiments 2-27, 2-29, 2-32 and 2-36. (a) Initial sample configuration with the compression (parallel to $x_{1}$ ) applied to the upper surface and simultaneous simple shear parallel to $x_{3}$. (b) Stage 1 samples have not reached minimum strain rates. The black diamond indicates where the sample was taken for thin-section examination. (c) The colour code showing the relative azimuth and plunge of the pixel distribution across a thin section that relates to the c-axis orientation. (d) Microstructure in experiment 2-27, illustrated as axial-distribution diagram. The shear strain $(\gamma)$ and shortening $(\varepsilon)$ for the experiment is shown below micrograph (on lower left corner). The lower-hemisphere equal-area projection shows the volume distribution of $c$ axes measured at a uniform spacing across a horizontal $(H)$ section through the centre of the sample. The histogram provides a quantitative measure of the c-axes characteristics. The curve (indicated by solid line) drawn along with the histogram represents a theoretical random crystal orientation. (e) Samples where the strain rate has increased to a tertiary creep value (stage 2). ( $f, g)$ Microstructures and c-axis distributions in horizontal sections. 


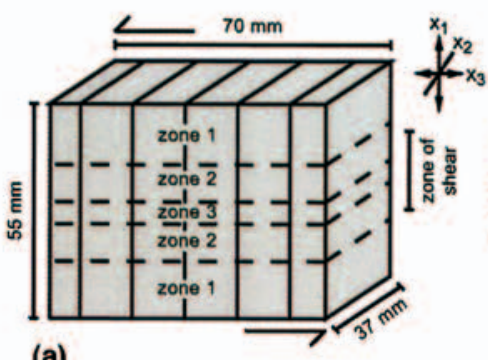

(a)

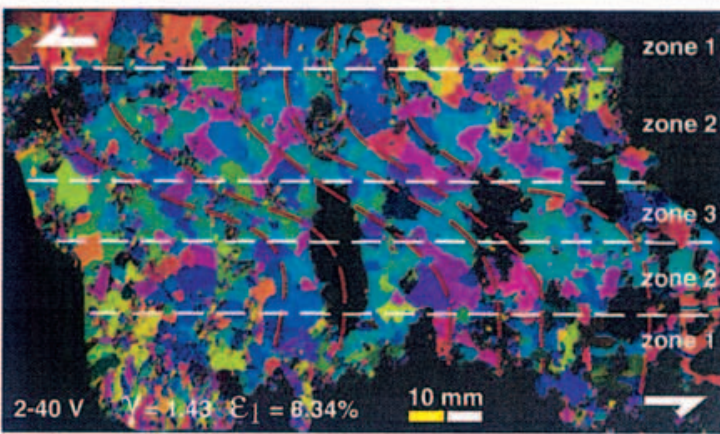

(c) Vertical section $\left(x_{1} x_{3}\right)$ experiment $2-40$
Initial layers parallel to $x_{1} x_{2}$ plane $\begin{array}{lll}\text { Compressive stress } \sigma & 0.22 \mathrm{MPa} \\ \text { Shear stress } & \tau & 0.4 \mathrm{MPa}\end{array}$ Temperature $\quad \mathrm{T} \quad-2^{\circ} \mathrm{C}$
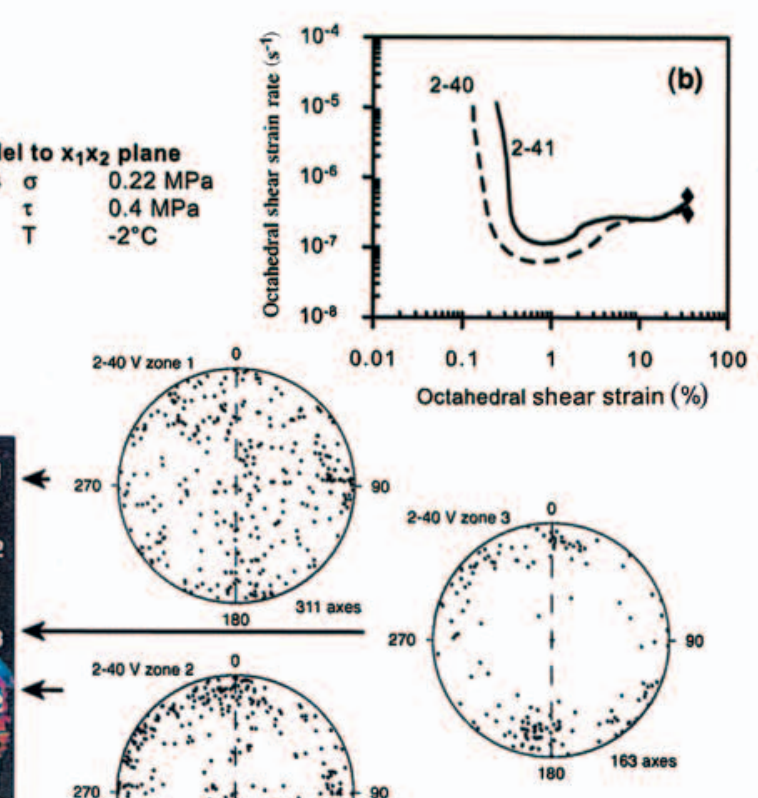

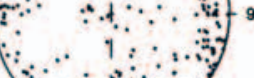

$180 \quad 397$ axes
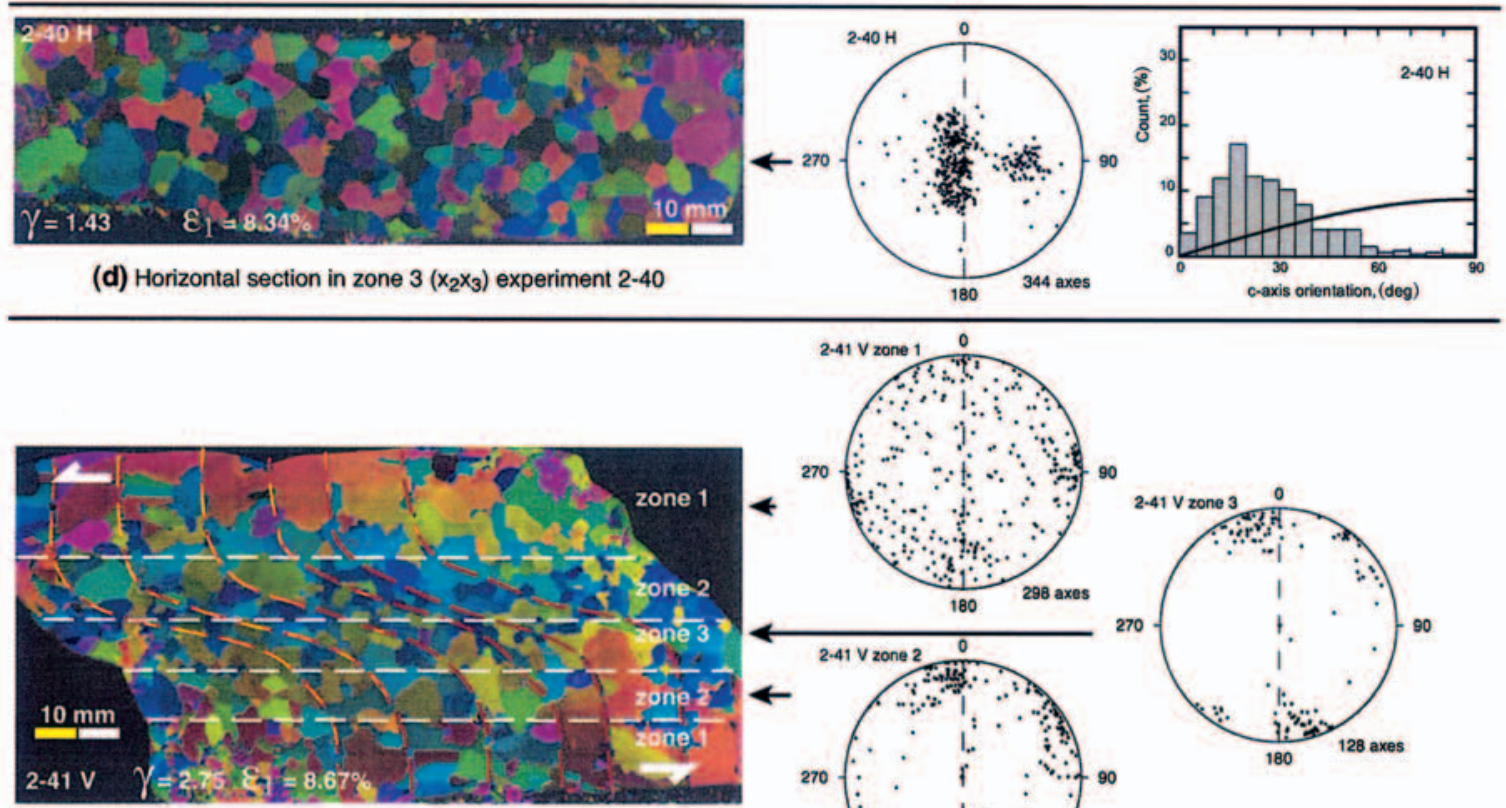

(e) Vertical section $\left(x_{1} x_{3}\right)$ experiment 2-41

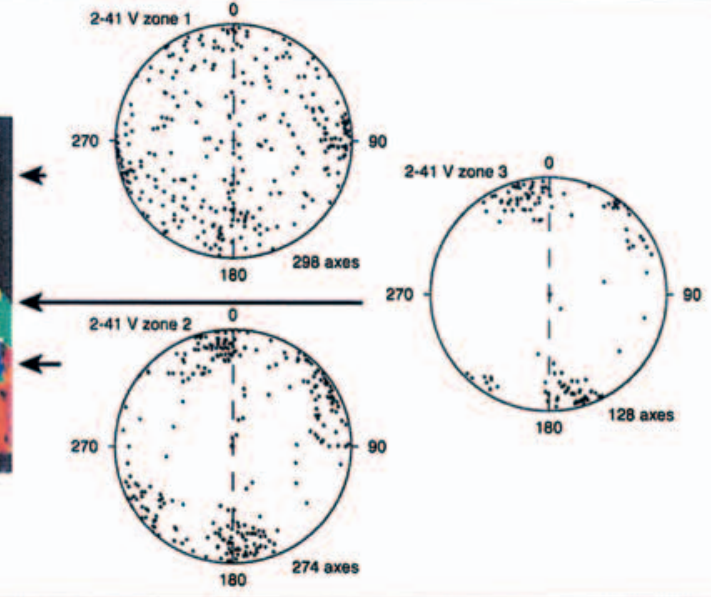

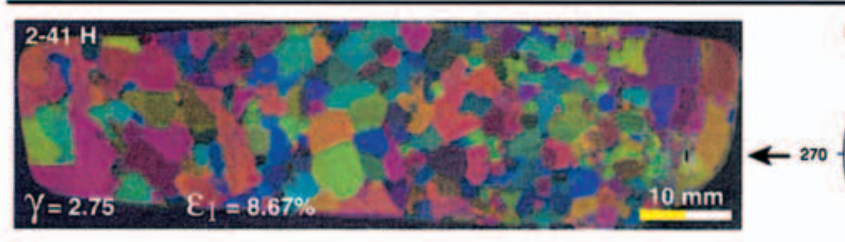

(f) Horizontal section in zone $3\left(x_{2} x_{3}\right)$ experiment 2-41
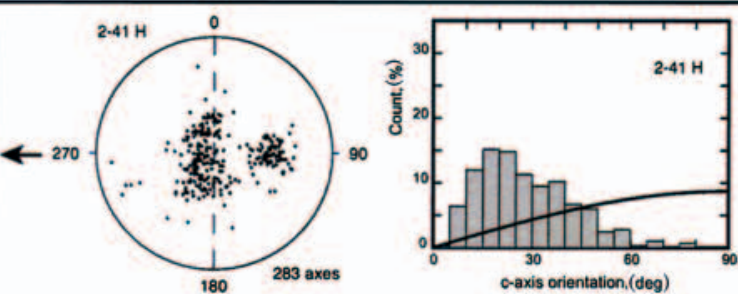

Fig. 4. Experiments 2-40 and 2-41 with layering initially parallel to the $x_{1} x_{2}$ plane. (a) Initial sample configuration. (b) Deformation creep curves for experiments. $(c-f)$ The microstructures illustrated in axial distribution diagrams (colour code is the same as in Figure $3 c$ and corresponds to the azimuths in the stereographic projections). The greatest degree of recrystallization occurs in zone 3, with zone 1 containing a high proportion of undeformed host grains. The corresponding lower-hemisphere equalarea projections show the distributions of c axes measured in horizontal $(H)$ and vertical $(V)$ sections through the centre of the deformed sample. The histograms show the deviation of the c axes from a random c-axis distribution.

first stage is where the $c$-axis fabric was measured when the strain rate was close to a minimum in different sets of experiments (e.g. Fig. 3b), which is on the transition point between an elastic and the onset of the plastic deformation.
Stage 1 is characterized by an inherited microstructure that reflects the original grain components, namely, large elongate grains and small interstitial grains. However, the elongate grains are sometimes undulose with subgrain 


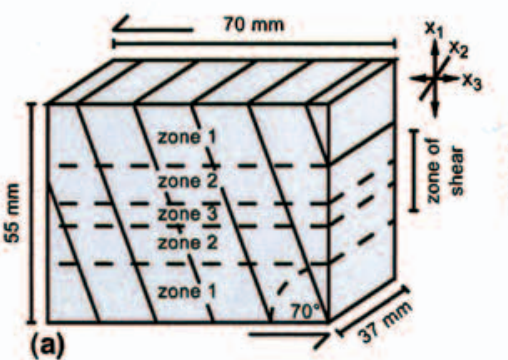

Initial layers $20^{\circ}$ to $x_{1} x_{2}$ plane

Compressive stress $\sigma \quad 0.22 \mathrm{MPa}$

Shear stress

Temperature

(a)

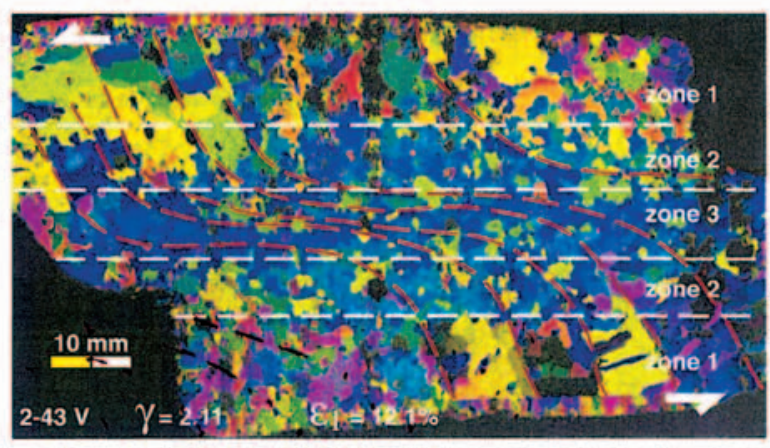

(c) Vertical section $\left(x_{1} x_{3}\right)$ experiment $2-43$
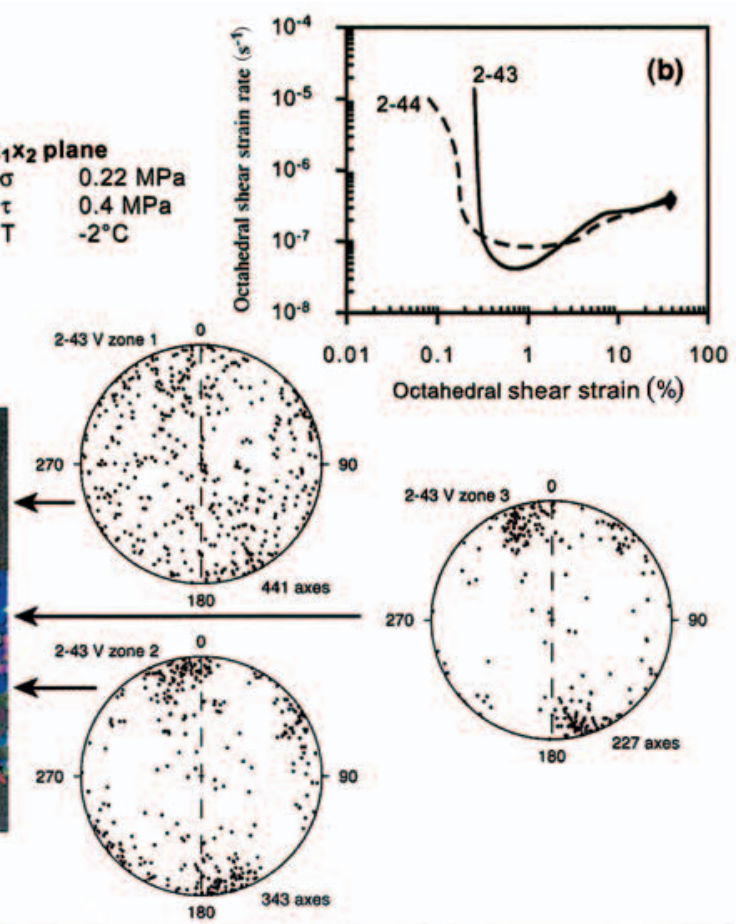
$\begin{array}{ll}\tau & 0.4 \mathrm{MPa} \\ \mathrm{T} & -2^{\circ} \mathrm{C}\end{array}$

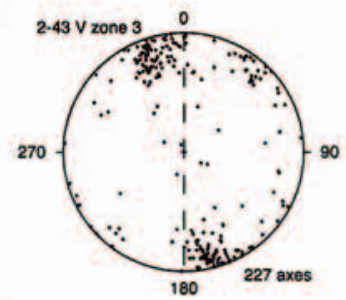

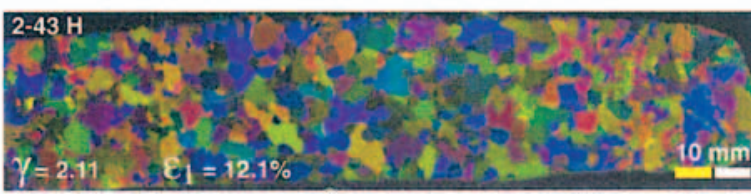

(d) Horizontal section in zone $3\left(x_{2} x_{3}\right)$ experiment 2-43
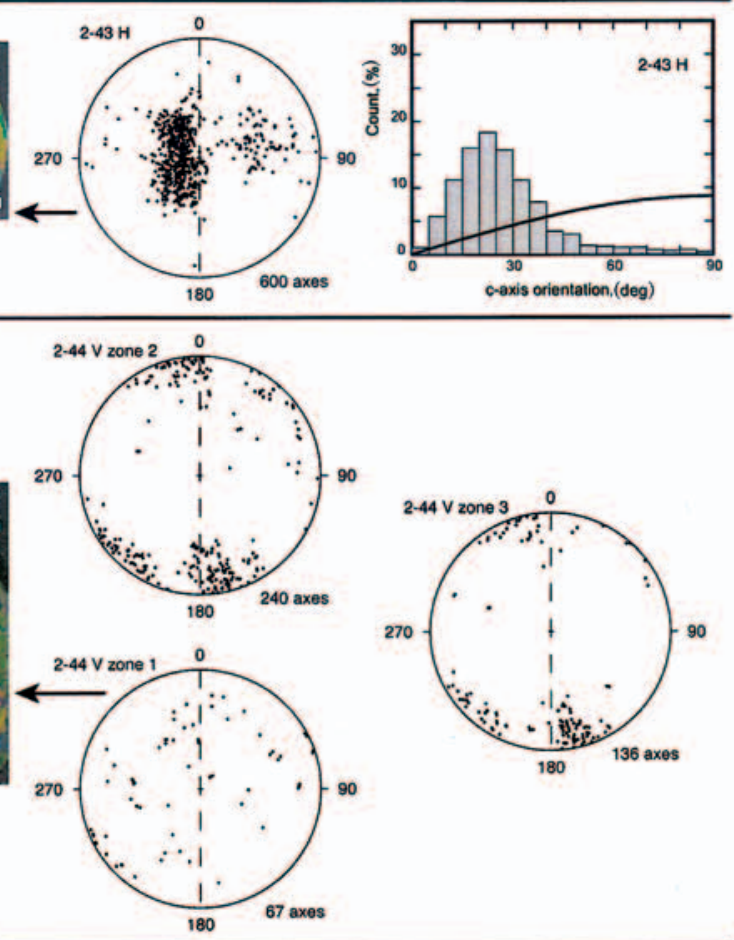

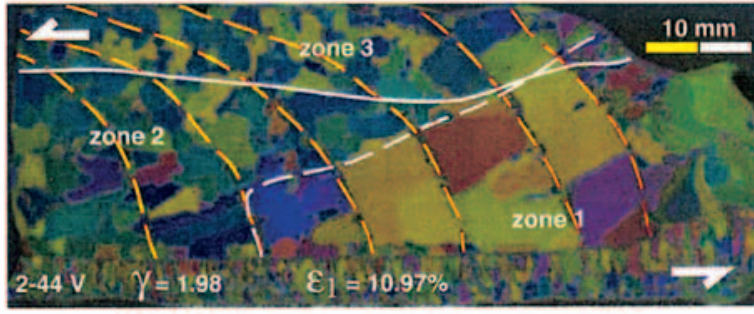

(e) A half vertical section $\left(x_{1} x_{3}\right)$ experiment $2-44$

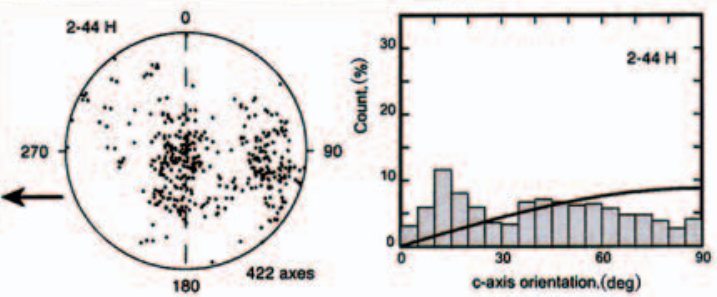

Fig. 5. Experiments 2-43 and 2-44 with layering initially inclined $20^{\circ}$ to the $x_{1} x_{2}$ plane. ( $\left.a-f\right)$ are explained in Figure 4 caption.

development, whereas grain boundaries of the small grains appear to have undergone migration (e.g. Fig. 3d; experiment 2-27). The associated patterns of preferred orientation, obtained from systematic $2 \mathrm{~mm}$ spaced measurements across the sample, still reflect the $c$-axis preferred orientation pattern that occurs in the starting material (Fig. 3d). This is a volume distribution of $c$ axes within the sample where the small grains have random $c$-axis orientation distributions and the coarse elongate grains have sub-horizontal concen- trations ( $c$ axes lying close to the $90^{\circ}$ azimuth in the stereographic projection in Fig. 3d). The inheritance of these $c$-axis concentrations is also supported by histograms of $c$-axis distributions during stage 1 (Fig. $3 \mathrm{~b}$ and d). These show a significant population of grains with $c$ axes lying at approximately $90^{\circ}$ to the layering that are above the theoretical random crystal orientation (Fig. 3d). These observations are comparable to those of Wilson and Russell-Head (1982) and Budd and Jacka (1989) who showed that initially isotropic ice 
remains isotropic through to the minimum strain rate at about $1-2 \%$ shortening strain but, with further deformation and recrystallization, gradually develops a clearly defined crystal fabric.

Once strain rate increases to a steady-state tertiary creep value (Fig. 3e), a distinct second stage (stage 2) in the microstructural and $c$-axis evolution develops (Fig. $3 f$ and $g$ ). New recrystallized grains replace the older grain population. The $c$-axis pattern seen in the histograms at the conclusion of these tests indicated the development of a new fabric. In the horizontal $x_{2} x_{3}$ sections, there is a suggestion of a girdle-like pattern that contains the compression axis while still retaining a strong concentration of $c$ axes sub-perpendicular to the layering (Fig. $3 \mathrm{f}$ and $\mathrm{g}$ ). Elements of this pattern are also observed in horizontal sections from more highly sheared regions (e.g. Fig. 4d and f; fabric diagrams 2-40 $\mathrm{H}$ and 2-41 $\mathrm{H})$. However, in the higher-strained samples (Fig. 4d and f), there are two asymmetric concentrations of $c$ axes relative to the compression axis with a strong single maximum, rotated with the sense of shear. There is considerable coalescence of similarly oriented recrystallized grains to produce a strengthening of the fabric. Regions of recrystallized grains (zone 3 in Fig. 4c and e) have the same orientation but are much larger than the recrystallized grains in the transitional region (zone 2 in Fig. 4c and e).

In vertical sections through high-strain zones (Fig. 4c, fabric diagram 2-40 V zone 3) the $c$-axis distributions still retain the strong concentration of $c$ axes preserved in the rotated layering. However, there is a discrete asymmetric distribution oblique to the compression axis, rotated slightly against the sense of shear with a new secondary maximum lying at $50^{\circ}$ to the dominant maximum (Fig. 4e, fabric diagram 2-41 V zone 3). This is also identified in the transitional zones (Fig. 4c and e, zone 2), whereas the relatively undeformed boundary zones (Fig. 4c and e, fabric diagrams 2-40 V zone 1; 2-41 V zone 1) preserve the initial $c$-axis fabric distribution. In general, the grains contributing to the new asymmetric pattern have $c$ axes that lie at high angles to the central shear zone.

Figure 5 illustrates samples where the initial layering was inclined to the shortening direction with random $c$-axis concentrations in the initial layering (Fig. 5c, fabric diagrams 2-43 zone 1). In horizontal sections (Fig. 5d and f), the stereographic projection displays $c$-axis concentrations that asymmetrically develop about the shortening direction, and a significant departure from a random distribution and the pattern seen in zone 1 . In vertical sections, specific asymmetric secondary-maxima concentrations can be identified. This can be clearly seen in the histograms that show the orientation distributions at different strains (Fig. $5 \mathrm{~d}$ and $\mathrm{f}$ ) and in the vertical sections (Fig. 5c and e, fabric diagrams 2-43 $\mathrm{V}$ zone 3; 2-44 $\mathrm{V}$ zone 3 ).

Figure 6 provides misorientation distribution data for four representative stages in the $c$-axis fabric development observed in sections cut parallel to the shear plane (horizontal sections) in experiments illustrated in Figures 3-5. Misorientation angle distributions are displayed using a histogram of the frequency of different misorientation angles between grain pairs. These data allow us to perform two kinds of misorientation analysis (Fig. 6): (1) a neighbour-pair misorientation calculated from two $c$-axis orientation measurements, on either side of a grain boundary between neighbouring grains, and (2) a random-pair misorientation calculated from the orientations of grain pairs that are not necessarily in physical contact (Fliervoet and others, 1999). The dataset sizes of the neighbour-pair and random-pair misorientation subsets are similar. In the case of randompair misorientation distribution, the measured $c$ axes of the neighbouring grain pairs are randomly paired using an Excel macro.

In the initial stages of deformation, where strain rate reaches a minimum (Fig. $3 \mathrm{~b}$ and d; stage 1) the neighbourpair misorientation distribution appears to reflect the starting material and appears to be essentially random (Fig. 6a), with a small population grouping at $80-90^{\circ}$. This grouping corresponds to the elongate grains that parallel the layering (the red-coloured grains in Fig. 3d), and reflects the predeformation orientation of these grains. At stage 2 of the deformation history, from the high-strain zone in the centre of the sample, the neighbour-pair misorientation $c$-axis distribution is non-random, characterized by a broad peak at $10-80^{\circ}$, with a sub-peak centred at $50-80^{\circ}$ (Fig. 6 b and c). The neighbour-pair misorientation distribution is very similar to the random-pair misorientation distribution and suggests that grains are not influenced by their neighbours. After the stage 2 deformation and in all high-strained samples, the $c$-axis pattern (Fig. $6 \mathrm{~d}$ ) and the neighbour-pair misorientation distribution is a broad peak at $10-90^{\circ}$ with sub-peaks at $10-30^{\circ}$ and $40-60^{\circ}$ (Fig. 6d). This two-maxima type fabric pattern can be observed in all samples where the octahedral shear strain exceeds $10 \%$, and is particularly obvious in the zones of greatest layer rotation (zone 3 in Figs $4 \mathrm{e}$ and $\mathrm{f}$ and $5 \mathrm{c}-\mathrm{f})$.

\section{FLOW RATE IN THE ANISOTROPIC ICE}

Of interest here is the combination involving shear strain with an orthogonal component of shortening and its effect on highly anisotropic ice. Representative creep curves for the current dataset are shown in Figures $3 \mathrm{~b}, 4 \mathrm{~b}$ and $5 \mathrm{~b}$ as plots of octahedral shear strain rate as a function of octahedral shear strain. These creep curves show that at $-2^{\circ} \mathrm{C}$ the octahedral strain rates for samples with layering parallel to the $x_{1} x_{2}$ plane (Fig. $3 \mathrm{~b}$ ) are very similar at low strains. At higher strains (Figs $4 \mathrm{~b}$ and 5b), there was not a uniform acceleration to a final steady-state creep as in the polycrystalline ice described by Li and Jacka (1998). Instead, shear strain rates increase and then decrease (e.g. experiment 236) before finally accelerating after octahedral shear strains of $<1 \%$ (e.g. Fig. 3e). This non-steady-state flow is accompanied by extensive recrystallization, and probably reflects the non-uniform behaviour between layers and the formation of a lenticular layering in the high-strained portions of the sample.

In samples initially inclined to the principal strain axes (Fig. 5), the strain-rate variation during the early stages of their initial strain history was more variable than in samples with layering parallel to the $x_{1} x_{2}$ plane. Once these samples had attained a minimum strain rate that varied from $\sim 4$ to $7.8 \times 10^{-8} \mathrm{~s}^{-1}$, they underwent a steady-state acceleration that was comparable to the results of $\mathrm{Li}$ and others (1996) for comparable compressive stresses. This acceleration can be correlated with the development of high shear strains within the sample, accompanied by extensive recrystallization. This suggests that the initial orientation of both the layering and the $c$ axes is critical in controlling the initial behaviour, but, with increasing strain, the strain-rate 

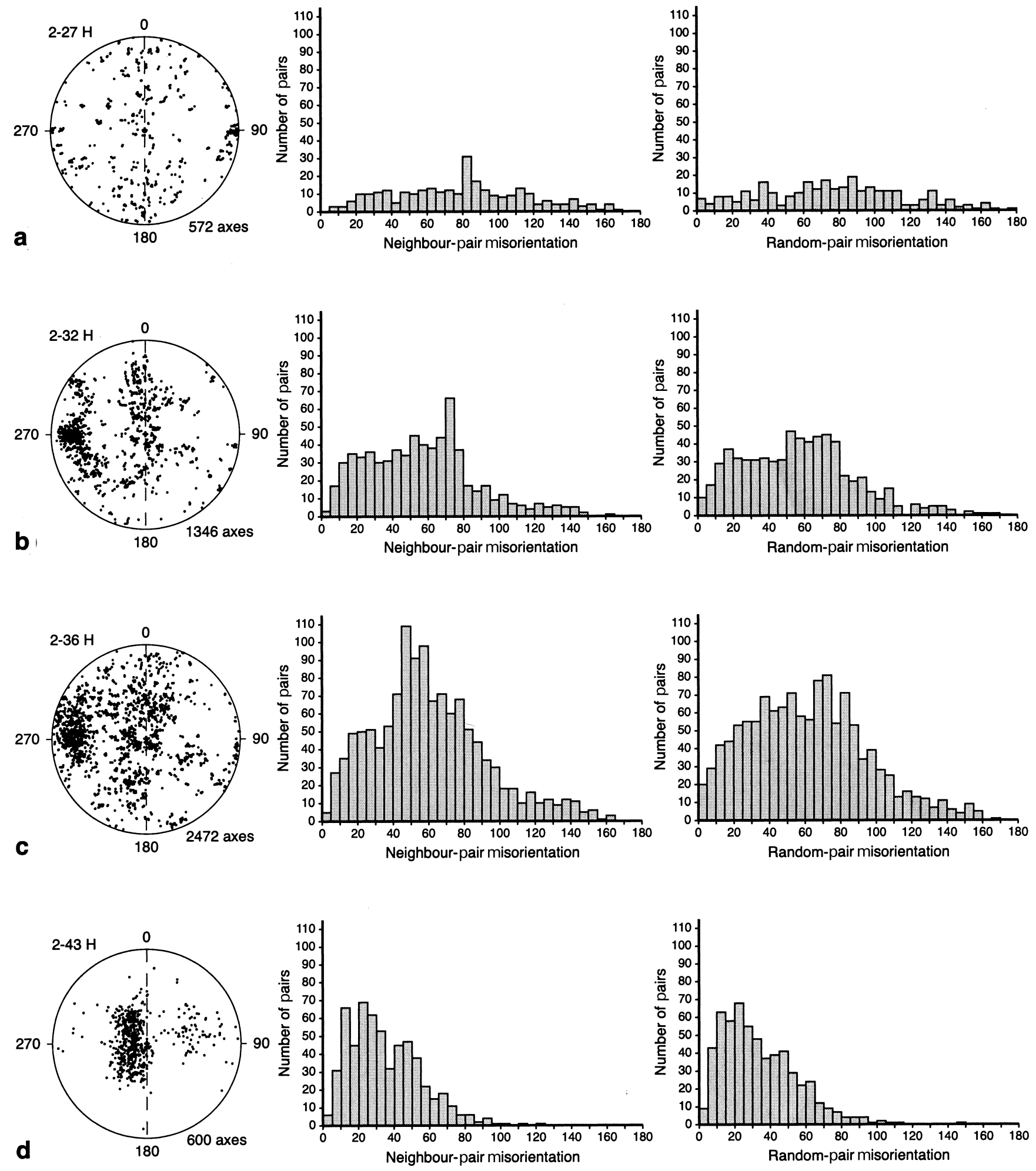

Fig. 6. Lower-hemisphere equal-area projection showing the distributions of c axes, and the corresponding neighbour-pair and random-pair misorientation distributions between adjacent grains measured in a horizontal (H) section within zone 3 of deformed samples 2-27 ( a), 2-32 (b), 2-36 (c) and 2-43 (d).

increases are similar to those in the other tests. The crystal fabric at the conclusion of these $-2{ }^{\circ} \mathrm{C}$ tests, again, is similar to the final fabric and final strain rates of $\sim 4 \times 10^{-7} \mathrm{~s}^{-1}$ in the vertically oriented samples.

\section{DISGUSSION AND GONGLUSIONS}

This experimental study was undertaken to characterize the strain, crystal-orientation fabrics and microstructures related to varying ice-layer orientations in a shear zone environment. Avariety of experimental configurations were used, in which strain is concentrated in zones parallel to a pre-existing anisotropy (Figs $1 \mathrm{a}-\mathrm{c}$ and 2), within passively folded layers (Fig. ld and e) and within compressional shear zones (Figs 3-5). Where deformation is localized to highstrain zones, the initial layering becomes lenticular and masked by extensive recrystallization (e.g. Fig. 5c); a new caxis fabric development and eventually grain growth obliterates the pre-existing fabric (e.g. Fig. 4e). In low-strain areas (Fig. 3), the $c$-axis preferred orientation is dominated by inherited fabric components from the host ice (Fig. 4, zone 1). At the onset of recrystallization, the $c$-axis preferred orientation display an asymmetric strong single maximum 
at a high angle to the plane of shear. This is rotated in the sense of shear and is accompanied by the development of a secondary $c$-axis maximum approximately $50^{\circ}$ to the shear plane. There is a strengthening of this crystallographic preferred orientation pattern with grain growth and the disappearance of any inherited fabric elements.

In these experiments there was a rapid transition from the girdle-like distribution of $c$ axis, which includes grains with inherited fabric elements, to a double-maxima orientation fabric that is developed in conjunction with the progression from minimum to maximum strain rates. This transition is important, because it probably corresponds to the situation in ice sheets where instabilities, such as folds with or without the development of a lenticular layering on the limbs, are propagated as simple shear begins to dominate over flattening to produce a non-coaxial strain regime (Alley and others 1997). Similarly, there is a lack of correlation between the layer dip angles and the respective mean $c$ axes as described by Tison and others (1994). The initial and minimum strain rates are highly dependent on the orientation of the stress with respect to the layering. This was also the conclusion of Castelnau and others (1998) who found the effect of misorientation of anisotropy by even a few degrees cannot be neglected when estimating the rheology of the ice in the ice sheet. However, the current investigation has shown that the mechanical response in an anisotropic ice mass can vary depending on the combination of stress regimes and the orientation of layering coupled with its crystallographic fabric.

\section{ACKNOWLEDGEMENTS}

Li Jun and T. H. Jacka from the Glaciology Group of the Australian Antarctic Division are thanked for their assistance with the construction of the combined shear and compression apparatus. D. S. Russell-Head is thanked for his development of the early versions of the "Microfabric Analyser" used to collect the $c$-axis data that formed the basis of this paper. Financial support from an Australian Research Grant is gratefully acknowledged. Two anonymous referees are thanked for a perceptive set of comments, which, along with the input of the scientific editor, S. J. Jones, greatly improved this paper.

\section{REFERENGES}

Alley, R. B., A. J. Gow, D. A. Meese, J. J. Fitzpatrick, E. D. Waddington and J.F. Bolzan. 1997. Grain-scale processes, folding and stratigraphic disturbance in the GISP2 ice core. F. Geophys. Res., 102 (C12), 26,819-26,830.

Bons, P. D., M.W. Jessell and C.W. Passchier. 1993. The analysis of progressive deformation in rock inclusions. F. Struct. Geol., 15 (3-5), 403-411.

Budd, W. F. and T. H. Jacka. 1989. A review of ice rheology for ice sheet modelling. Cold Reg. Sci. Technol., 16(2), 107-144.

Castelnau, O. and 7 others. 1998. Anisotropic behavior of GRIP ices and flow in central Greenland. Earth Planet. Sci. Lett., 154(1-4), 307-322.

Fliervoet, T. F., M. R. Drury and P. N. Chopra. 1999. Crystallographic preferred orientations and misorientations in some olivine rocks deformed by diffusion and dislocation creep. Tectonophysics, 303(1-4), 1-27.

Glasser, N. F., M. J. Hambrey, K. R. Crawford, M. R. Bennett and D. Huddart. 1998. The structural glaciology of Kongsvegen, Svalbard, and its role in landform genesis. F. Glaciol., 44(146), 136-148. (Erratum: 46(154), 2000, p. 538.)

Gold, L.W. 1972. The process of failure of columnar-grained ice. Philos. Mag., 26(2), 311-328.

Hambrey, M. J. 1977. Foliation, minor folds and strain in glacier ice. Tectonophysics, 39(1-3), 397-416.

Hudleston, P. J. 1980. The progressive development of inhomogeneous shear and crystallographic fabric in glacial ice. F. Struct. Geol., 2 (1-2), 189-196.

Jacka, T. H. and M. Maccagnan. 1984. Ice crystallographic and strain rate changes with strain in compression and extension. Cold Reg. Sci. Technol., $\mathbf{8}(3), 269-286$.

Li Jun and T. H. Jacka. 1998. Correspondence. Horizontal shear rate of ice initially exhibiting vertical compression fabrics. F. Glaciol., 44(148), 670-672.

Li Jun, T. H. Jacka and W.F. Budd. 1996. Deformation rates in combined compression and shear for ice which is initially isotropic and after the development of strong anisotropy. Ann. Glaciol., 23, 247-252.

Lisle, R. J. 1985. Geological strain analysis: a manual for the $\mathrm{Rf} / \phi$ technique. Oxford, Pergamon.

Marmo, B. A. and C. J. L. Wilson. 1998. Strain localisation and incremental deformation within ice masses, Framnes Mountains, east Antarctica. $\mathcal{F}$. Struct. Geol., 20(2-3), 149-162.

Tison, J.-L., T. Thorsteinsson, R. D. Lorrain and J. Kipfstuhl. 1994. Origin and development of textures and fabrics in basal ice at Summit, central Greenland. Earth Planet. Sci. Lett., 125 (3-4), 421-437.

Tison, J.-L., R. D. Lorrain, A. Bouzette, M. Dini, A. Bondesan and M. Stiévenard. 1998. Linking landfast sea ice variability to marine ice accretion at Hells Gate Ice Shelf, Ross Sea. In Jeffries, M. O., ed. Antarctic sea ice: physical processes, interactions and variability. Washington, DC, American Geophysical Union, 375-407. (Antarctic Research Series 74.)

Twiss, R. J. and E. M. Moores. 1992. Structural geology. New York, W. H. Freeman and Company.

Wilson, C. J. L. 1981. Experimental folding and fabric development in multilayered ice. Tectonophysics, 78(1-4), 139-159.

Wilson, C. J. L. 2000. Experimental work on the effect of pre-existing anisotropy on fabric development in glaciers. In Maltman, A. J., B. Hubbard and M. J. Hambrey, eds. Deformation of glacial materials. London, Geological Society, 97-113. (Special Publication 176.)

Wilson, C. J. L. and D. S. Russell-Head. 1982. Steady-state preferred orientation of ice deformed in plane strain at $-1{ }^{\circ} \mathrm{C}$. F. Glaciol., 28 (98), 145-160.

Wilson, C. J. L., D. S. Russell-Head and H. M. Sim. In press. The application of an automated fabric analyzer system to the textural evolution of folded ice layers in shear zones. Ann. Glaciol., 37. 